Ann. Sci. forest., 1981, 38 (1), 3-30

\title{
Comportement de jeunes plants d'Epicéa commun en sol à engorgement temporaire de surface : influence de divers facteurs du milieu
}

\author{
G. LEVY \\ Avec la collaboration technique de C. Brechet, Y. LefÈvre \\ Station de Recherches sur les Sols forestiers et la Fertilisation \\ I.N.R.A., \\ Centre de Recherches de Nancy \\ Champenoux, 54280 Seichamps
}

\section{Résumé}

Cette étude fait la synthèse de plusieurs expériences effectuées en milieu contrôlé, sur des plants repiqués à 2 ans, l'engorgement étant réalisé généralement l'année même du repiquage. Le substrat est prélevé dans des stations hydromorphes de l'Est de la France assez riches sur le plan chimique.

L'influence de différents facteurs sur le taux de mortalité et la croissance des plants a été précisée, notamment : durée de l'engorgement, température, potentiel d'oxydo-réduction de la nappe, caractéristiques du sol.

Il a été montré que le déficit hydrique des parties aériennes constituait la cause directe de leur mortalité en sol engorgé sur le substrat utilisé. Ainsi, une nébulisation très fréquente d'eau sur les feuilles fait pratiquement cesser toute mortalité. Le ressuyage de plants longtemps engorgés provoque une accélération du dépérissement, probablement parce qu'il supprime une certaine alimentation passive en eau.

Les possibilités d'approvisionnement en air dans la rhizosphère conditionnent l'aspect des anciennes racines ainsi que la densité des racines nouvelles. Le taux final de survie est lié à ces caractéristiques du système racinaire, ainsi qu'à l'apparition d'organes qui peuvent être considérés comme des adaptations à l'hydromorphie, en particulier, au niveau du collet des plants, des lenticelles fortement hypertrophiées.

L'engorgement entraîne un important déficit en azote dans les aiguilles, débutant très peu de temps après l'installation de la nappe, même si celle-ci est relativement oxygénée. La teneur foliaire en manganèse est également fortement affectée. Des déficits en phosphore et potassium n'apparaissent que plus tardivement.

11 est apparu que la carence azotée créée par la nappe constitue une des causes primaires de la mortalité des plants. Un déficit du sol en phosphore agit dans le même sens, mais d'une façon moindre. Des pulvérisations d'azote sur les aiguilles durant l'engorgement sont beaucoup plus efficaces si elles sont effectuées à l'aide d'acides aminés qu'avec de l'ammonitrate ou de l'urée.

\section{1. - Introduction}

L'Epicéa commun (Picea abies L.) est une espèce très utilisée en reboisement des sols à hydromorphie temporaire, notamment dans l'Est de la France. Les résultats sont souvent bons, mais ce n'est pas toujours le cas (DEcourt \& LEVY, 1974); ils 
dépendent des fluctuations de la nappe, mais aussi des conditions de station (LEVY, 1978). Il est cependant impossible de déterminer dans une étude de terrain l'importance réelle de chacun dee facteurs en cause : plusieurs d'entre eux varient simultanément d'une placette à l'autre et, de plus, on assiste à une fluctuation continuelle du niveau des nappes. Aussi avons-nous effectué des expériences en serre sur de jeunes plants, en contrôlant un certain nombre de facteurs. Nous avions ainsi précédemment (LEVY, 1971) comparé les conséquences de différents niveaux de nappe.

Nous examinerons ici le cas de l'engorgement de surface, en faisant varier successivement la durée de cet engorgement, l'époque où il intervient, les teneurs en eau du sol après ressuyage, certaines propriétés du sol, l'oxygénation de la nappe. Nous indiquerons l'influence de ces facteurs sur la mortalité des plants, leur croissance aérienne, leur enracinement et leur nutrition minérale, sans attacher trop d'importance à certaines valeurs absolues en raison des conditions microclimatiques particulières qui règnent dans une serre. L'effet néfaste de facteurs tels une nappe à faible potentiel d'oxydo-réduction ou une forte température extérieure sur beaucoup de plantes de culture est connu; nous avons voulu préciser ce qu'il en était dans le cas de l'Epicéa.

Nous avons aussi essayé, par ailleurs, de comprendre un peu mieux certains des faits observés. On peut ainsi se demander quel phénomène est directement à l'origine de la mort des plants, ou quelle influence les perturbations nutritives ou hormonales créées par l'engorgement ont sur la mortalité ou la baisse de croissance. Il s'agira en grande partie d'un exposé de synthèse qui reprend les résultats de plusieurs expériences successives.

\section{2. - Matériel et méthode}

Les expériences dont nous présentons les résultats se sont déroulées en serre plusieurs années de suite, et elles se complètent mutuellement. Nous considérerons ces expériences d'une façon synthétique, en groupant l'ensemble des résultats par thème et en donnant des exemples tirés, chaque fois, de l'une quelconque des expériences concernées par ce thème (seules des différences significatives ont été citées dans ces exemples).

Nous avons toujours utilisé des plants d'Epicéa repiqués à 2 ans, en serre, dans des vases de végétation cylindriques et d'environ $30 \mathrm{~cm}$ de hauteur et de diamètre (en général, 9 plants par vase); le fond de ces vases est garni d'une hauteur de $5 \mathrm{~cm}$ de gravillons au-dessus desquels se trouve le sol. Un piézomètre perforé de fentes horizontales permet les apports d'eau; le niveau de l'eau est maintenu à la hauteur voulue grâce à un tuyau en plastique souple dont une extrémité, percée de petits orifices sur une vingtaine de centimètres de long, repose horizontalement au fond du vase; le tuyau ressort du vase à cet endroit, et il est maintenu verticalement près de sa paroi extérieure jusqu'au niveau désiré, c'est-à-dire, dans ces expériences-ci, la surface du sol. Les vases de végétation sont disposés sur de grandes tablettes pouvant chacune en contenir 60. Il s'agit dans tous les cas de dispositifs en blocs, dont la délimitation tient compte le plus possible des gradients microclimatiques de la serre. 
Nous avons étudié le rôle d'un certain nombre de facteurs en les faisant varier un à un à partir des condlitions de «base» suivantes : engorgement de surface au cours de l'année-même du repiquage après le temps nécessaire à l'installation des plants, pendant un minimum de 28 jours (et un maximum de 3 mois) et débutant au printemps lorsque la nouvelle pousse terminale des plants atteint en moyenne $1 \mathrm{~cm}$ de long, absence de fertilisation ou dapport quelconque, pas de renouvellement de l'eau, le sol ćtant formé en général de 6 à $8 \mathrm{~cm}$ d'un mull mésotrophe assez riche à texture limoneuse $\left(\mathrm{P}_{2} \mathrm{O}_{\mathrm{i}}=0,11 \mathrm{p} .1000, \mathrm{C} / \mathrm{N}=14, \mathrm{~S} / \mathrm{T}=61\right.$, prélevé en Lorraine dans un sol à pseudogley) qui surmonte un horizon $\mathrm{A}_{2 .}$ limoneux (prélevé dans le même sol) ou un sable grossier; après ressuyage, le sol ést maintenu à unc humidité en moyenne un peu inférieure à la capacité au champ.

Les résultats que nous présenterons correspondent à ces conditions de base, qui ne seront ainsi plus précisées, sauf pour le facteur que l’on fera varier.

Certaines de ces interventions nécessitent des précisions :

- Trois méthodes différentes ont été utilisées pour améliorer le potentiel d'oxydoréduction du milieu par rapport à une nappe stagnante; nous espérions ainsi obtenir une représentation de diverses conditions pouvant exister dans le milieu naturel :

- insufflation permanente d'air à partir du fond des vases de végétation, à l'aide de pompes d'aquarium;

- renouvellement fréquent de l'eau d'engorgement;

- apports de $\mathrm{NO}_{3}$ (oxydant) : $50 \mathrm{ml}$ de solution de nitrate de calcium à $152 \mathrm{~g} / 1$ à 3 reprises, réparties au cours de la durée de l'engorgement. Des apports plus fréquents ou à doses supérieures fournissent pratiquement les mêmes résultats.

- Afin que le système racinaire des plants soit placé dans les conditions les moins défavorables possibles, nous avons effectué dans certains traitements, après habillage approprié, un repiquage à racines presque horizontales, c'est-à-dire disposées dans les $2-3 \mathrm{~cm}$ de surface du sol, où la nappe est en contact avec l'atmosphère libre.

- Des apports d'éléments nutritifs au sol ont été effectués à deux reprises, à une dizaine de jours d'intervalle, le second précédant l'engorgement de quelques jours; nous avons ensuite «lavé»le sol à plusieurs reprises, afin notamment d'éviter que la nappe n'ait une forte pression osmotique. Les seaux non engorgés ont été "lavés » dans les mêmes conditions. Les doses suivantes ont été appliquées à chaque reprise :

- $\mathrm{N}: 3$ g d’ammonitrates à 34 p. 100 de $\mathrm{N}$;

- PK : $50 \mathrm{ml}$ de solution de $\mathrm{PO}_{4} \mathrm{H}_{2} \mathrm{~K}$ à $40 \mathrm{~g} / \mathrm{l}$;

- P : $50 \mathrm{ml}$ de solution de $\mathrm{Ca}\left(\mathrm{PO}_{4} \mathrm{H}_{2}\right)_{2} \mathrm{H}_{2} \mathrm{O}$ à $40 \mathrm{~g} / \mathrm{l}$.

Cette fertilisation préalable est destinée à procurer des réserves nutritives aux plants, car l'absorption des éléments minéraux risque d'être perturbée durant l'engorgement (HEller, 1969).

- En raison de cette dernière probabilité, des apports d'azote ont également été effectués par pulvérisation foliaire pendant la période d'engorgement. 
Il s'agit d'applications successives (souvent toutes les semaines, parfois une fois par quinzaine) des produits suivants :

- urée : solutions à 5 à $9 \mathrm{~g} / 1$;

- ammonitrates à 34 p. 100 : solution à $2 \mathrm{~g} / 1$;

- acides aminés : solution à $1 \mathrm{~g} / 1$ en tout, comprenant les produits suivants : acide $\mathrm{L}$ glutamique, $\mathrm{L}$ glutamine, $\mathrm{L}$ asparagine, acide $\mathrm{L}$ aspartique, $\mathrm{L}$ arginine, $\mathrm{L}$ leucine, $\mathrm{L}$ alanine, $\mathrm{L}$ proline.

On a aussi pulvérisé dans les mêmes conditions des produits hormonaux, dont l'engorgement réduit la synthèse (REID \& RAILTON, 1974) :

- benzyladénine : solution à $50 \mathrm{mg} / \mathrm{l}$;

- acide gibbérelique : solution à $250 \mathrm{mg} / \mathrm{l}$.

Pour toutes ces pulvérisations, on a joint à la solution un agent mouillant, le tween (les témoins ont reçu aussi la pulvérisation d'une solution de tween), on a protégé dans la mesure du possible le sol des produits pulvérisés, et on a lavé le feuillage le lendemain à l'aide d'eau pure afin d'éliminer éventuellement les dépôts de précipitation.

- Nous avons retenu la mesure du potentiel d'oxydo-réduction (Eh) de préférence à celle de la teneur en $\mathrm{O}_{2}$ pour caractériser les propriétés de la nappe vis-à-vis des racines, car elle prend également en compte les propriétés réductrices du milieu après disparition de l'oxygène. La mesure de Eh est cependant peu sensible et pas très fidèle; elle permet néanmoins de différencier quantitativement des nappes à propriétés nettement différentes, à condition d'effectuer les mesures à un même moment, car Eh varie assez fortement dans le temps et en fonction de la température du milieu. Par ailleurs, une mesure de Eh, statique, ne peut sans doute pas rendre entièrement compte des possibilités de renouvellement (par diffusion) de $\mathrm{O}_{2}$ au contact des racines.

\section{3. - Résultats et commentaires}

\section{1. - La mortalité des plants}

L'Epicéa est une espèce qui résiste généralement bien à l'engorgement. Des mortalités se produisent néanmoins dans les reboisements, en particulier au cours des mois qui suivent la plantation (cas auquel on peut comparer nos conditions expérimentales de base).

En serre, un engorgement dans les conditions de base décrites précédemment entraîne, s'il se poursuit assez longtemps, une mortalité presque générale, qui peut d'ailleurs ne se réaliser en grande partie qu'après le ressuyage. La première manifestation du dépérissement est, au cours de l'engorgement, un flétrissement des nouvelles pousses, qui retombent latéralement.

\subsection{1. - Durée de l'engorgement. Période de ressuyage}

La durée d'engorgement à partir de laquelle quelques plants meurent dépend des conditions climatiques, ensoleillement en particulier, qui conditionnent beaucoup 
la température dans la serre, bien que celle-ci soit partiellement climatisée. Elle varie généralement entre 12 et 20 ou 25 jours. Quelques jours (5 à 10) d'engorgement supplémentaires entraînent à terme une mortalité affectant la grande majorité des plants.

Une période de ressuyage interrompant l'engorgement est très favorable à la survie des plants. Ainsi, dans une expérience où 28 jours d'engorgement provoquaient à terme un taux de mortalité de 97 p. 100, un ressuyage de 7 jours séparant cet engorgement en deux périodes de 14 jours chacune permettait la survie finale de 23 p. 100 des plants.

\subsection{2. - Etat d'avancement phénologique lors de l'engorgement}

Si la longueur des pousses de l'année est plus faible lors de l'engorgement, les conséquences en sont moins défavorables. Ainsi, 43 jours d'engorgement ont permis la survie à terme de 3 p. 100 des plants dont la pousse terminale de l'année avait une longueur d'environ 1 à $2 \mathrm{~cm}$ lors de l'engorgement et de 21 p. 100 des plants repiqués 11 jours plus tard que les précédents et qui venaient seulement de débourrer lorsque la nappe a été installée. De même, si la pousse terminale de l'année est supérieure à $2 \mathrm{~cm}$ lors de l'engorgement, la mortalité apparaît plus précocement et évolue plus rapidement. On suppose que la vitesse de croissance est plus grande lorsque la pousse de l'année a déjà une certaine longueur; les besoins des plantes en oxygène sont alors plus élevés et les effets de la nappe plus défavorables.

Si le débourrement n'a pas encore eu lieu lors de l'engorgement, il ne se produit en général pas, et le plant se maintient vivant (il «végète») au moins jusqu'à l'automne; son devenir est néanmoins incertain.

\subsection{3. - Plants installés depuis un an ou plus}

\section{Influence des conditions hydriques au cours de l'été}

Des plants installés depuis un an ou plus résistent beaucoup mieux à la présence d'une nappe ; un engorgement de 28 jours n'a ainsi entraîné, sur de tels plants, aucune mortalité, quel que soit le stade d'application. Une certaine mortalité apparaît à partir d'un engorgement de 35 jours.

D'autre part, contrairement à ce que l'on constate si l'on installe la nappe l'année même du repiquage, l'engorgement n'empêche jamais le débourrement du bourgeon terminal, bien qu'il le retarde parfois un peu.

Nous avions précédemment étudié l'influence des conditions hydriques régnant dans le sol après suppression de la nappe sur des plants engorgés l'année même du repiquage (LEVY, 1971); la nappe n'affleurait cependant pas en surface, ce qui aurait fait périr la plupart des plants. Nous avons ici complété cette précédente étude en opérant sur des plants installés depuis plus d'un an et qui ont subi une nappe superficielle pendant 42 jours. Les résultats sont représentés sur la figure 1. On peut constater que la mortalité ne se produit que si la grande majorité des plants ont débourré à la fin de la période d'engorgement; elle est alors beaucoup plus importante si l'on maintient une assez forte humidité dans le sol (nappe à $-14 \mathrm{~cm}$ en l'occurrence) après suppression de la nappe de surface que si on laisse le sol se dessécher assez fortement (jusqu'à $\mathrm{pF}$ de 4,0). Ce résultat est sarıs doute lié au développement du système racinaire, nous en reparlerons ultérieur,ment. 


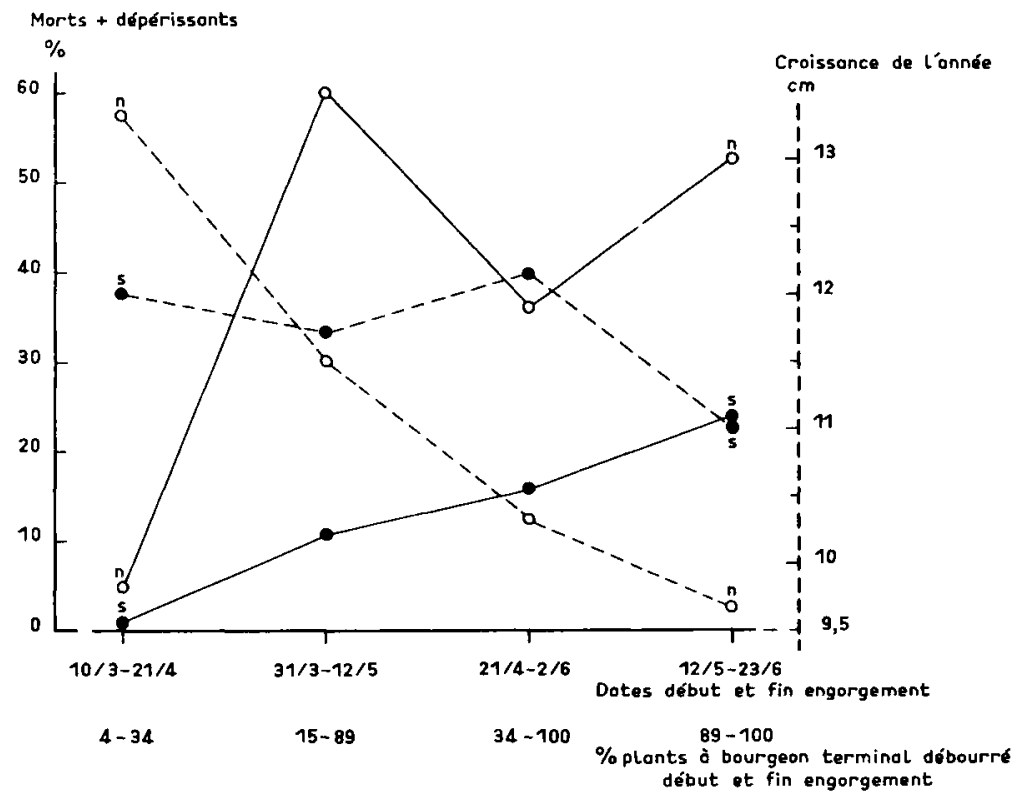

Fig. 1

Mortalité et croissance en fonction de l'époque de l'engorgement et du traitement après ressuyage (plants installés depuis plus d'un an)

Mortality and growth according to water logging time and to the treatment after water-table removing (on transplanted since more than one year plants)

Légende :

Traitement après ressuyage :

$\mathrm{s}$ : dessèchement relatif du sol,

$\mathrm{n}$ : nappe à $-14 \mathrm{~cm}$.

Treatment after water-table removing :

$\mathrm{s}$ : relative soil drying,

$\mathrm{n}:$ water-table at $-14 \mathrm{~cm}$.

Au cours de l'expérience citée ci-dessus (Levy, 1971), un fort desséchement du sol en été avait provoqué une mortalité plus importante que dans le cas que nous venons d'évoquer; il s'agissait de plants qui avaient subi une nappe à $-7 \mathrm{~cm}$ durant le printemps. Mais ces plants étaient moins bien installés et contenaient probabiement moins de réserves nutritives, car l'engorgement avait débuté peu de temps après le repiquage.

\subsection{4. - La température ambiante}

Nous avons réalisé simultanément un engorgement de surface dans deux groupes de vases de végétation installés dans deux serres différentes; la survie finale a été de 41 p. 100 des plants dans la serre la moins chaude, de 24 p. 100 dans l'autre. Cette influence défavorable d'une augmentation de la température s'explique sans doute par une aggravation du déficit en oxygène, car les besoins en cet élément sont alors accrus, alors que le potentiel d'oxydo-réduction s'abaisse; l'alimentation des plants en eau est donc encore plus mal assurée (cf. § 3.17). 


\subsection{5. - Texture et matière organique du sol}

Nous citerons, à titre d'exemple, les résultats globaux d'un dispositif :

\begin{tabular}{|c|c|c|c|}
\hline & $\begin{array}{l}A_{1}(8 \mathrm{~cm}) \\
+ \text { sables grossiers }\end{array}$ & $\begin{array}{l}A_{1}(8 \mathrm{~cm}) \\
+ \text { limons }\end{array}$ & $A_{1} \operatorname{pur}(25 \mathrm{~cm})$ \\
\hline Survie finale $(\%) \ldots \ldots \ldots$ & 41 & 12 & 3 \\
\hline $\begin{array}{l}\text { Eh à }-10 \mathrm{~cm} \text { (à un mo- } \\
\text { ment donné) }(\mathrm{mv}) \ldots \ldots \ldots\end{array}$ & +65 & +15 & -55 \\
\hline
\end{tabular}

On constate ( 2 premières colonnes) l'influence favorable sur la survie des plants d'une texture grossière, probablement par l'intermédiaire d'un meilleur potentiel d'oxydo-réduction dans la rhizosphère. Nous avons par ailleurs noté que la texture argileuse était la plus défavorable de toutes.

D'autres expériences nous ont montré que la matière organique peut avoir des conséquences variées sur la survie des plants. Ainsi, un horizon $\mathrm{A}_{1}$ est néfaste lorsqu'il surmonte un substrat minéral formé de sables grossier (survie de 30 p. 100 en l'absence d' $A_{1}, 16$ p. 100 avec $A_{1}$ ), utile quand ce substrat minéral est limoneux (survie de 4 p. 100 en l'absence d' $A_{1}, 24$ p. 100 avec $A_{1}$ ). Dans ce dernier cas (substrat limoneux), en l'absence d'horizon $A_{1}$ les limons deviennent très battants et se tassent beaucoup, alors qu'un horizon $A_{1}$ permet le maintien d'une certaine structure en surface, ce qui favorise la diffusion de l'oxygène dans le milieu : cet effet prend alors le pas sur l'abaissement du potentiel redox provoqué par la matière organique.

Par contre, une épaisseur trop importante d'horizon organique est défavorable, même en présence d'un substrat minéral limoneux (cf. $2^{\circ}$ et $3^{\circ}$ colonnes du tableau ci-dessus).

La matière organique a donc un effet défavorable sur la survie des plants lorsque la baisse du potentiel redox qu'elle provoque n'est pas compensée par une nette amélioration de la structure dans les premiers centimètres du sol.

\subsection{6. - Amélioration du potentiel d'oxydo-réduction au niveau des racines}

La présence d'une nappe dans la zone d'enracinement est toujours défavorable, même si elle est relativement oxygénée : en effet, le coefficient de diffusion de $\mathrm{O}_{2}$ est d'environ 300000 fois plus faible dans l'eau que dans l'air.

Cela étant, on peut penser que les propriétés de la nappe vis-à-vis des racines sont fonction de son potentiel redox. Celui-ci est déterminé par les propriétés du sol (texture, matière organique), mais aussi, en forêt, par les conditions de station, notamment la situation géographique et topographique : le renouvellement de l'eau, son oxygénation, en dépendent. Aussi avons-nous amélioré artificiellement le potentiel redox de la nappe, afin d'observer les conséquences d'un engorgement par une nappe moins défavorable théoriquement que celle que nous avons considérée jusqu'ici. 

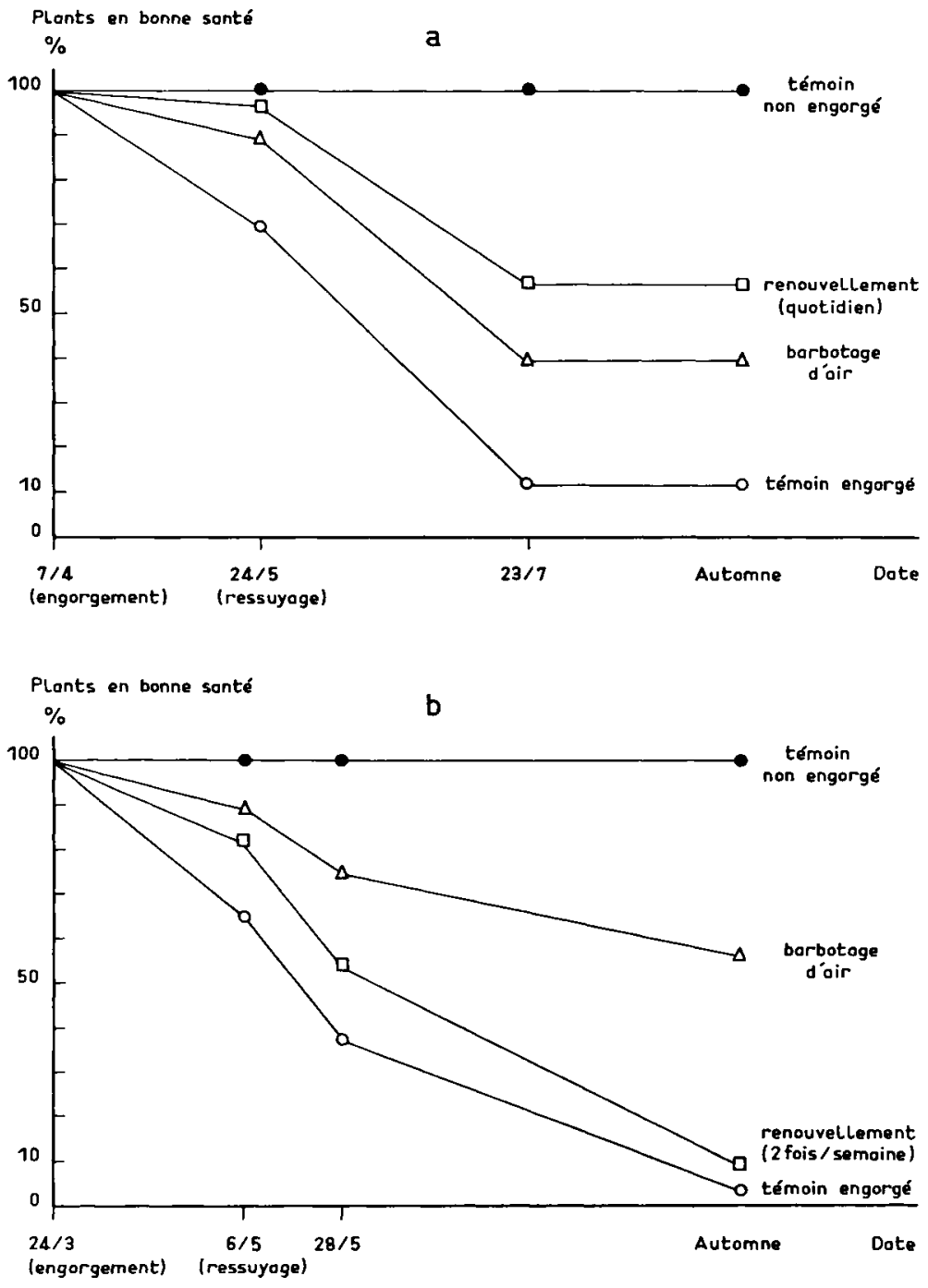

FIG. 2

Evolution du dépérissement

Dying evolution

Barbotage permanent d'air dans le vase ou renouvellement fréquent de l'eau Ces deux opérations améliorent la survie.

Ainsi sur sable pur, après un engorgement de printemps, la survie finale est passée de 57 p. 100 à 92 p. 100 grâce à une insufflation d'air.

Sur un substrat comprenant un horizon $A_{1}$ surmontant un sable, deux expériences (fig. 2) ont montré l'influence favorable de ces deux traitements, le renouvel- 
lement quotidien de l'eau (fig. 2 a) étant beaucoup plus efficace qu'un renouvellement effectué seulement deux fois par semaine (fig. 2 b). Signalons que le chiffre définitif de mortalité est en fait atteint en moyenne un peu moins de deux mois après le ressuyage, quel que soit le traitement.

Ces résultats sont probablement en rapport avec l'état d'oxydo-réduction du milieu : la mesure du potentiel redox, bien que n'étant guère discriminante dans les premiers temps de l'engorgement, permet par la suite de séparer le témoin engorgé et le traitement à renouvellement de l'eau; ainsi, sur même substrat que celui des expériences correspondant à la fig. 2, Eh était respectivement de +30 et $+125 \mathrm{mv}$ après un mois environ d'engorgement et de -125 et $+35 \mathrm{mv}$ deux semaines plus tard. Par contre, sa mesure dans les vases subissant un barbotage d'air fournit des résultats très peu fidèles, sans doute parce que les bulles d'air créent dans le milieu une discontinuité perturbant le passage du courant.

\section{Apport de $\mathrm{NO}_{3}^{-}$durant l'engorgement}

Comme les interventions précédentes, l'apport de $\mathrm{NO}_{3}-$ augmente le potentiel redox au niveau des racines; par exemple, après 5 semaines d'engorgement, Eh est de $+65 \mathrm{mv}$ contre $-175 \mathrm{mv}$ pour le témoin engorgé. Cependant, si très souvent cet apport ne modifie pas sensiblement la teneur des aiguilles en azote, on note dans certains cas un accroissement non négligeable de cette concentration; aussi ne peut-on toujours attribuer la totalité de l'amélioration de la survie à une augmentation du potentiel redox.

L'apport de $\mathrm{NO}_{3}{ }^{-}$abaisse en effet le taux de mortalité. Ainsi, effectué à deux reprises au cours de l'engorgement d'un sol à horizon $A_{1}$ surmontant un sable, il a fait passer la mortalité de 76 p. 100, pour le témoin engorgé, à 41 p. 100 ; dans les vases de végétation où l'on avait pris la précaution de changer l'eau environ deux jours après l'apport de $\mathrm{NO}_{3}{ }^{-}$afin d'éviter une nette augmentation de la pression osmotique du milieu, cette mortalité n'était plus que de 18 p. 100, alors que ce renouvellement de l'eau n'avait pas modifié la survie du témoin engorgé.

Cependant, si l'engorgement se poursuit assez longtemps (par exemple 2 mois), la mortalité finale est pratiquement totale, même si l'on effectue périodiquement d'autres apports de $\mathrm{NO}_{3}{ }^{-}$. Cela n'était pas le cas lorsqu'on renouvelait quotidiennement l'eau du vase : cette dernière intervention est la plus efficace parmi celles tendant à éviter des conditions trop défavorables au niveau des racines.

\section{Racines «horizontales»}

Ce traitement est également très favorable à la survie. L'apparition et l'évolution de la mortalité y sont nettement moins rapides que pour le témoin engorgé. Nous avons ainsi noté, une semaine après le ressuyage (l'engorgement ayant duré 1,5 mois), 30 p. 100 de plants morts au lieu de 62 p. 100 et, 2 semaines plus tard, respectivement 50 et 76 p. 100 de plants morts ; la mortalité est cependant totale après un engorgement suffisamment long. Il paraît évident que ces différences entre traitements sont la conséquence des conditions d'oxydo-réduction régnant dans les deux cas au niveau du système racinaire. 


\subsection{Facteur directement responsable de la mort des plants}

Les facteurs du milieu semblent donc intervenir sur le taux de survie principalement en influençant le potentiel redox au niveau du système racinaire ; nous examinerons ainsi plus loin leurs conséquences sur la morphologie de ce dernier. En ce qui concerne son fonctionnement, il est sans doute très perturbé (phénomène signalé pour de nombreux végétaux) car le milieu est hypoxique ou même anoxique : l'énergie dont les racines disposent est fortement diminuée; de plus, des perturbations d'ordre métabolique (nature et quantité des produits terminaux) affectent les espèces non ou peu tolérantes (Hook et Scholtens, 1978).

Quant à la cause directe de la mortalité des parties aériennes (sur substrats peu organiques et chimiquement assez riches sur lesquels se trouvent les pseudogleys qui nous intéressent), plusieurs explications peuvent être avancées, entre autres : intoxication par absorption d'un excès de certains corps formés en milieu réducteur ; carence sévère en certains éléments minéraux; déficit hydrique provoqué non seulement par le mauvais état du système racinaire, mais aussi par la baisse de perméabilité des tissus racinaires à l'eau quand l'oxygène fait défaut (Kramer, 1969). (Ces trois phénomènes sont souvent signalés comme conséquences de l'engorgement). Il semble qu'on puisse rejeter les deux premières hypothèses, car nous avons trouvé des compositions foliaires analogues dans le témoin engorgé et dans certains traitements où la survie était au contraire assez élevée, tant pour les éléments nutritifs principaux que pour certains éléments qui peuvent être toxiques ( $\mathrm{Fe}, \mathrm{Mn}, \mathrm{S}, \mathrm{Al}$ ). Cela ne constitue pas une preuve indéniable, néanmoins nous avons voulu tester préférentiellement la $3^{\text {e }}$ hypothèse. Trois phénomènes tendent à l'accréditer :

\section{Mortalité et ressuyage}

Nous avons indiqué précédemment qu'à la suite d'un engorgement suffisamment long pour provoquer une mortalité très importante, celle-ci se manifeste souvent surtout après le ressuyage (suivi d'un maintien du sol à la capacité au champ); or, si au lieu de ressuyer, on maintient la nappe encore quelque temps, le flétrissement des plants est sensiblement retardé, bien que finalement, et c'est normal, la mortalité rattrape puis dépasse celle des plants ressuyés plus tôt. La figure 3 illustre ce phénomène ; elle est relative à une expérience dans laquelle la seule différence de traitement concerne la date de ressuyage : les valeurs relevées le $21 / 9$ représentent pratiquement le taux de survie final.

On peut ainsi supposer qu'après une certaine durée d'engorgement, la présence de la nappe permet encore pendant quelque temps une certaine absorption passive d'eau, qui ne serait plus possible si l'on ressuie. Cela suggère que la mortalité des plants consécutive à la présence d'une nappe superficielle est bien provoquée directement par un déficit hydrique interne.

\section{Bilan hydrique des plants}

L'hypothèse est renforcée par les résultats d'analyses de la teneur en eau moyenne, lors du ressuyage, des aiguilles de plants soumis à divers traitements. Nous avons ainsi relevé les chiffres suivants (humidité en p. 100 de poids sec) : absence de nappe : 231 p. 100 , nappe avec barbotage d'air ou apport de $\mathrm{NO}_{3}: 210$ p. 100, témoin engorgé vert : 152 p. 100, témoin engorgé jaunissant : 74 p. 100, valeurs qui sont bien dans le même ordre que le taux de survie final. 


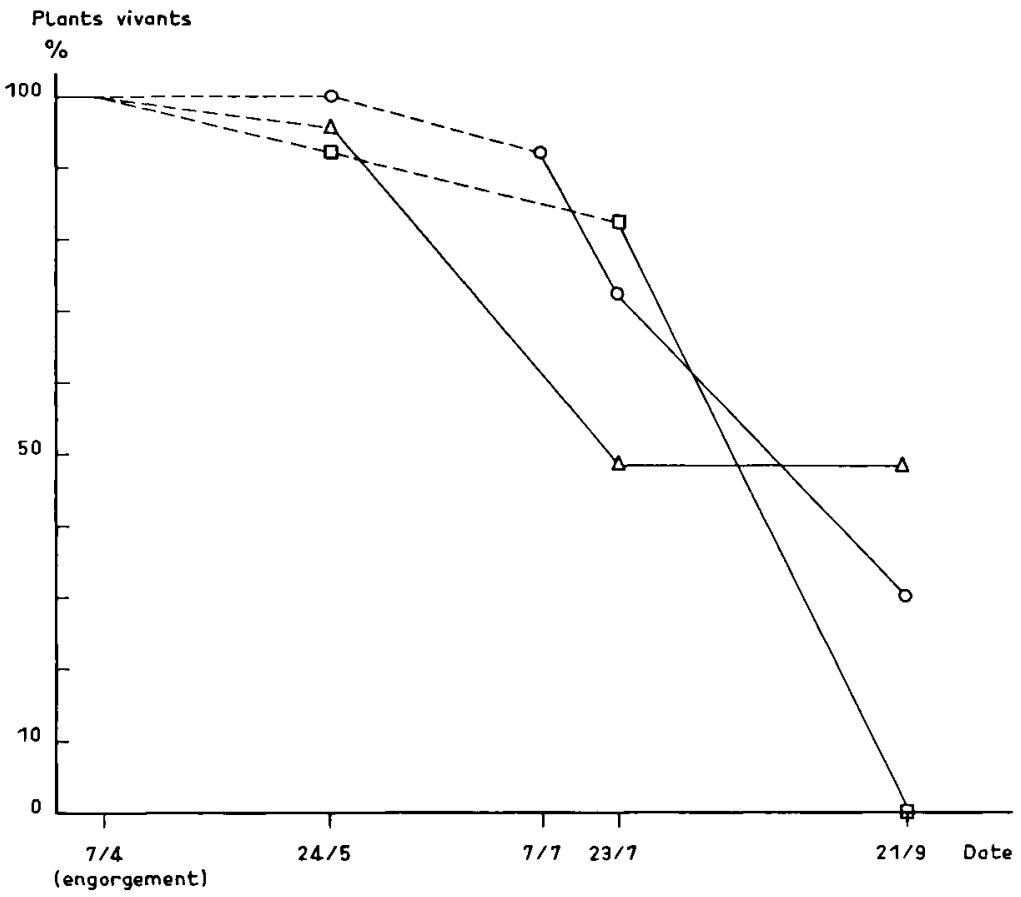

FIG. 3

Influence de la date de ressuyage sur l'évolution de la mortalité (sol engorgé, avec barbotage d'air)

Influence of water-table removing date on mortality evolution (water logged soil, with air bubbling)

Légende :

Engorgement de surface.

Surface water-logged soil.

Sol ressuyé.

Soil without water-table.

\section{Essai de protection contre l'établissement du déficit hydrique interne}

Nous avons enfin tenté de vérifier s'il était possible de conserver en vie des plants qu'une longue période d'engorgement devait en principe faire périr, en maintenant artificiellement une teneur en eau élevée dans les parties aériennes. Nous avons ainsi appliqué une nébulisation fréquente (30 secondes par heure) qui réduit beaucoup la transpiration foliaire et permet l'absorption d'eau par les aiguilles. La figure 4 représente l'évolution de la survic avec le temps en fonction de l'application ou non de la nébulisation et de la période au cours de laquelle celle-ci est maintenue. Il apparaît que cette technique permet de diminuer considérablement, voire de supprimer presque complètement, la mortalité, que ce soit au cours de l'engorgement ou après le ressuyage; mais son efficacité cesse dès qu'on l'arrête : la nébulisation remplace les racines dans leur rôle de maintien du potentiel hydrique dans les parties aériennes, mais évidemment n'améliore pas l'état ni le fonctionnement du système racinaire. 


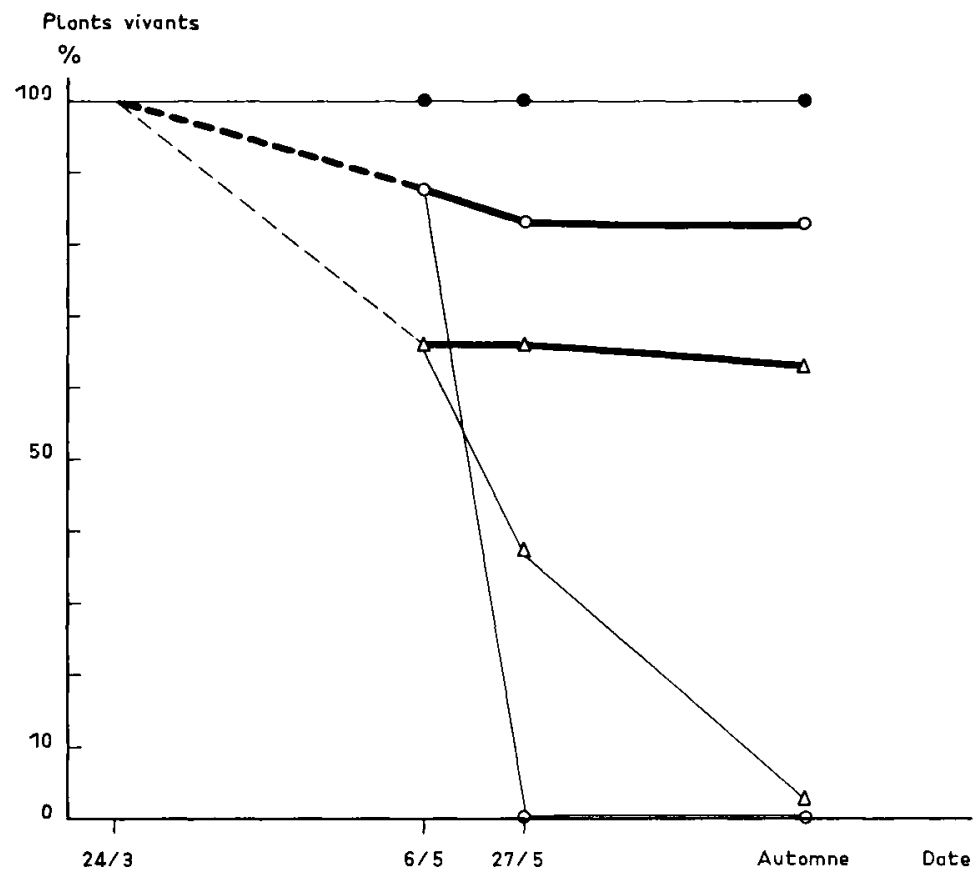

FIG. 4

Influence de la nébulisation sur la survie des plants

Influence of water spraying on plants survival

Légende :

_ _ _ Sol engorgé ; pas de nébulisation.

Water-logged soil ; no water spraying.

- - Sol engorgé ; nébulisation.

Water-logged soil ; water spraying.

Sol ressuyé ; pas de nébulisation.

Soil without water-table; no water spraying.

Sol ressuyé ; nébulisation.

Soil without water-table; water spraying.

L'ensemble de ces résultats confirme donc l'hypothèse selon laquelle la mortalité est provoquée directement par un déficit hydrique. Certaines objections à cette conclusion pourraient cependant être émises :

- La nébulisation appliquée au cours de l'engorgement diminue l'E.T.P. au niveau des aiguilles; l'absorption d'eau, donc de produits toxiques réducteurs contenus dans la nappe, est donc réduite (ce qui irait dans le sens de l'hypothèse : mortalité provoquée par intoxication). Mais le fait que la nébulisation appliquée seulement lors du ressuyage fasse cesser toute mortalité (fig. 4) supprime cette objection ; cette application permet de compenser l'abaissement moyen de teneur en eau des aiguilles mentionné au paragraphe précédent : cet abaissement était donc bien à l'origine de la mortalité qui allait suivre, en l'absence de nébulisation. 
- L'apport fréquent en surface du vase de végétation, par nébulisation, d'eau sans doute à teneur assez élevée en oxygène crée des conditions de milieu moins défavorables au niveau des racines, ce qui pourrait expliquer la baisse de mortalité constatée. Cette hypothèse n'est en fait pas non plus à retenir : en effet, d'une part, la nébulisation est efficace, comme il vient d'être indiqué, même si elle n'est appliquée qu'après ressuyage; d'autre part, si une nébulisation appliquée dès l'engorgement est supprimée au ressuyage, cela provoque une mortalité foudroyante (cf. fig. 4) : les conditions de milieu au niveau des racines étaient donc bien très défavorables; et enfin, des mesures ont montré que la nébulisation en cours d'engorgement n'augmentait pratiquement pas le potentiel redox au niveau des racines.

\section{2. - La croissance aérienne}

Nous avons montré précédemment que la production de l'Epicéa adulte (LeVY, 1978) et la croissance de jeunes plants (LeVY, 1972) étaient d'autant plus faibles que la nappe était plus superficielle; elles dépendent aussi du potentiel redox de la nappe, lequel est très lié à la texture du sol.

En serre, d'une manière très générale, les facteurs dont dépend la mortalité plus ou moins grande agissent aussi sur la croissance aérienne des plants. Celle-ci est d'ailleurs très affectée par un engorgement de surface, surtout s'il intervient l'année même du repiquage, même dans des conditions nettement moins drastiques que celles qui entraînent une mortalité. Tout comme pour la mortalité, des différences de croissance par rapport au témoin se manifestent déjà en cours d'engorgement, puis elles se poursuivent et s'amplifient souvent après le ressuyage, principalement en raison de la disparition de la pousse d'août dans les traitements engorgés.

\section{Durée de lengorgement. Période de ressuyage. Etat d'avancement phénologique}

Trois jours d'engorgement en surface suffisent souvent à affecter quelque peu la croissance aérienne. Un ressuyage interrompant pendant quelques jours l'engorgement est très bénéfique, comme pour la survie; dans l'expérience citée précédemment à ce propos ( 28 jours d'engorgement continus ou interrompus par 7 jours de ressuyage), la pousse terminale de l'année avait, à la fín de la période d'engorgement du second traitement, $43 \mathrm{~mm}$ de long dans un cas et $54 \mathrm{~mm}$ dans l'autre (n'ont été évidemment mesurés que les plants encore vivants).

Contrairement à ce que l'on constate pour la survie, la croissance de la pousse terminale au cours de l'engorgement (pour des plants repiqués au cours de la même année) est souvent d'autant plus importante que cette pousse était plus grande au moment de l'installation de la nappe; ce résultat est probablement dû au fait que l'engorgement intervient alors à un stade phénologique où la vitesse de croissance est plus élevée. De même, comme nous le mentionnions précédemment, si le bourgeon terminal n'est pas débourré lors de l'engorgement, il ne débourre en général pas cette année-là. Mais de toute façon la pousse des plants engorgés par une nappe stagnante non oxygénée est très faible.

Plants plus âgés

Les résultats sont fort différents si l'on opère sur des plants installés depuis au moins un an sans avoir subi d'engorgement. La présence d'une nappe n'empêche 
alors pas le débourrement du bourgeon terminal. Seule risque d'être affectée la croissance de plants qui subissent un engorgement d'une certaine durée alors que le débourrement s'est déjà produit.

Les conditions hydriques régnant dans le sol après suppression de la nappe interviennent sur la longueur de la pousse annuelle, et il y a interaction avec l'époque de l'engorgement, tout comme pour la survie. Ainsi, pour l'expérience citée à propos de la mortalité (cf. fig. 1), un sol maintenu bien humide (traitement $\mathrm{n}=$ nappe à - $14 \mathrm{~cm}$ ) est favorable à la croissance aérienne, par comparaison avec l'autre traitement d' «été » ( $\mathrm{s}=$ sol desséché et maintenu à un $\mathrm{pF}$ voisin de 4,0 ) lorsque l'engorgement s'était produit avant le débourrement du bourgeon terminal de la plupart des plants (fig. 1, $1^{\text {re }}$ date). Par contre, ce même traitement $n$ est d'autant plus défavorable que l'engorgement de surface avait été appliqué plus tardivement (dates 2 à 4). Nous reviendrons à cette expérience à propos de l'enracinement.

On peut s'étonner, par ailleurs, de l'absence de différence marquée de croissance de l'année pour les diverses dates d'engorgement, dans le cas du traitement d'été s; on suppose que si l'engorgement est par lui-même plus néfaste lorsqu'il intervient aux dates les plus tardives, cet inconvénient est compensé par le fait que les plants survivants de ces traitements subissent moins longtemps la sécheresse qui fait suite.

\section{Influence du potenticl d'oxydo-réduction}

Les changements du potentiel redox de la nappe au niveau des racines, quelle qu'en soit la cause, modifient la croissance des plants au cours de l'engorgement, tout comme le taux de mortalité, mais en général dans une bien moindre mesure : des plants engorgés l'année-même du repiquage poussent en général peu, quelles que soient les conditions; de plus, certains facteurs qui étaient favorables durant la présence de la nappe peuvent au contraire ralentir la croissance après le ressuyage, ou vice-versa : ainsi en est-il de la texture (par exemple sables, par rapport à des limons) ou de la matière organique (horizon $A_{1}$ ) ; cela n'est évidemment pas le cas des interventions qui améliorent le potentiel redox dans un substrat donné (insufflation d'air, renouvellement fréquent de l'eau, apport de $\mathrm{NO}_{3}{ }^{-}$) : elles restent favorables à la croissance, même après le ressuyage : à titre d'exemple, dans une expérience citée à propos de la mortalité ( $A_{1}$ sur sable), les moyennes des pousses terminales à la fin de l'engorgement sont de $6,9 \mathrm{~cm}$ pour le témoin non engorgé, de $4,2 \mathrm{~cm}$ pour le traitement à barbotage d'air, et de $1,8 \mathrm{~cm}$ pour le témoin engorgé.

Par ailleurs, dans le cas de plants dont le bourgeon terminal est presque débourré lors de l'engorgement, une amélioration des conditions d'oxydo-réduction, par rapport à la nappe stagnante, favorise fortement le débourrement. Ainsi, dans une expérience (sur substrat : $\mathrm{A}_{1}+$ sable), trois apports de $\mathrm{NO}_{3}{ }^{-}$au cours des 43 jours d'engorgement ont permis de faire baisser de 20 à 7 p. 100 le taux de plants non débourrés.

\section{Nébulisation d'eau sur les feuilles}

Cette intervention améliore quelque peu la croissance des plants engorgés. Ainsi, dans l'expérience citée ci-dessus, dans laquelle la pousse du témoin engorgé était de $1,8 \mathrm{~cm}$, la nébulisation a amené cette pousse à $3,1 \mathrm{~cm}$ : l'engorgement de surface provoque donc sans doute, à certains moments, des déficits hydriques internes, même sur des plants qui ne sont pas destinés à mourir. Ce déficit en eau serait une des causes (mais pas la plus importante) de la chute de croissance aérienne des plants. 
Par ailleurs, cette même nébulisation fréquente, appliquée à des traitements non engorgés, augmente fortement la teneur en eau du sol et diminue d'autant sa porosité remplie d'air. La croissance des plants en est quelque peu affectée : pousse terminale de $5,7 \mathrm{~cm}$ au lieu de $6,9 \mathrm{~cm}$. Donc, même en l'absence de nappe, une teneur du sol en eau supérieure à la capacité au champ est défavorable.

\section{3. - La nutrition minérale lors de l'engorgement de surface}

\subsection{1. - Influence de l'engorgement de surface sur l'état nutritif des plants}

Une alimentation minérale normale nécessite, chez la plupart des végétaux, une bonne aération des racines (HELleR, 1969). Mais qu'en est-il exactement pour l'Epicéa dans le cas d'un engorgement de surface?

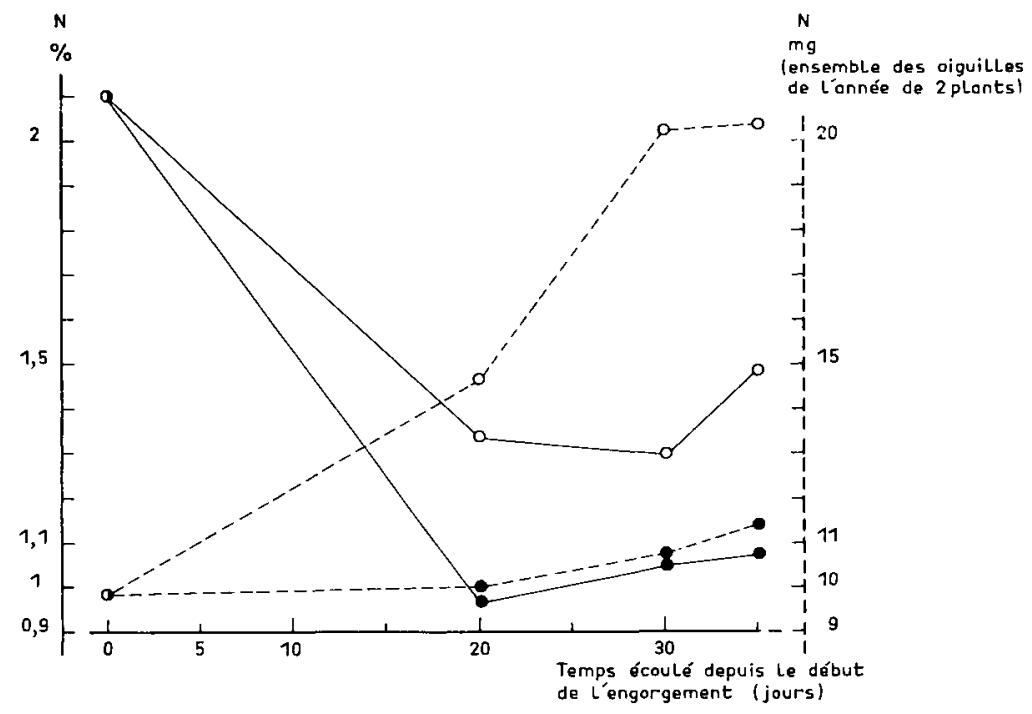

FIG. 5

Influence de l'engorgement de surface sur l'azote des aiguilles Influence of surface water logging on needles nitrogen

Légende :

O Sol non engorgé.

Soil without water-table.

- Sol engorgé.

Soil with water-table.

Rappelons que chez l'Epicéa adulte, un fort engorgement de printemps entraîne une carence azotée dans les feuilles en automne (LEVy, 1978). 
En serre, le phénomène le plus net, du moins si l'engorgement se produit l'année même du repiquage, est également un important déficit en azote des aiguilles de l'année; ce déficit est déjà décelable peu de temps après la mise en place de la nappe (7 jours par exemple); il semblerait même qu'il n'y ait aucune assimilation de cet élément en présence d'une nappe superficielle (fig. 5). Le poids total d'azote dans l'ensemble des aiguilles de l'année est en fait très variable selon les plants; pour avoir une certitude, il faudrait examiner l'évolution pour un même plant, ce qui est évidemment impossible (ou utiliser de l'azote marqué). Les petites différences notées sur la fig. 5 entre le $20^{\circ}$ et les $30^{\circ}$ et $35^{\circ}$ jours, pour les plants ennoyés, ne sont pas significatives.

Une amélioration du potentiel redox ne modifie guère la teneur foliaire en azote : dans l'expérience correspondant à la figure 5, les plants qui bénéficiaient d'un barbotage d'air dans la nappe contenaient dans leurs aiguilles 1,09 p. 100 de $\mathrm{N}$ au $20^{\circ}$ jour d'engorgement et 0,96 p. 100 au $30^{\circ}$ jour. Cette valeur n'évolue plus sensiblement ensuite, jusqu'à la fin de l'engorgement, alors que la concentration foliaire en azote du témoin non engorgé remonte jusqu'à plus de 2,0 p. 100.

Le renouvellement quotidien de l'eau fournit pratiquement les mêmes résultats que le barbotage d'air.

L'engorgement de surface a des conséquences beaucoup moins marquées en ce qui concerne les deux autres éléments nutritifs principaux, P et $\mathbf{K}$. Leur concentration dans les aiguilles ne diffère jamais significativement de celle du témoin non engorgé au moins jusqu'au $21^{\circ}$ jour d'engorgement. Un déficit net est apparu après une durée variable selon les expériences, et les résultats sont pratiquement les mêmes quelque soit le potentiel redox de la nappe : pour $\mathrm{P}$ entre le $40^{\circ}$ et le $60^{\circ}$ jour d'engorgement, pour $\mathrm{K}$ entre le $22^{\circ}$ et le $70^{\circ}$ jour, environ. Les figures 6 et 7 indiquent, à titre d'exemple, l'évolution de la concentration foliaire en $\mathbf{P}$ et $\mathbf{K}$ dans la même expérience que celle représentée sur la figure 5; les valeurs relatives au témoin engorgé après le 30" jour n'ont pas été mentionnées, car elles correspondent vraisemblablement à des plants pour la plupart en «sursis».

L'examen de l'évolution des teneurs totales (en $\mathrm{mg}$ ) en $\mathrm{P}$ et $\mathrm{K}$ dans les aiguilles de l'année (très variables, comme dans le cas de $\mathrm{N}$, selon les plants) laisse supposer que, contrairement à $\mathrm{N}$, ces 2 éléments sont assimilés au cours des premiers temps de l'engorgement, au moins pendant 3 semaines. Cependant, certaines difficultés d'assimilation existent peut-être à ce moment; elles seraient alors masquées (le pourcentage foliaire en $P$ et $K$ ne les ferait pas apparaître) par la carence azotée, qui constitue le facteur limitant essentiel de la croissance sur le plan nutritif, et éventuellement par des phénomènes de translocation interne.

Les plants qui, après ressuyage vers la fin du printemps, sont encore vivants (et non dépérissants) à l'automne ont alors en général une concentration foliaire en $N$, $P$ et $K$ voisine de celle du témoin non engorgé. Il s'avère donc qu'une analyse foliaire d'automne ne permet pas toujours de diagnostiquer un fort engorgement de printemps.

Par ailleurs, et sur un autre plan, l'engorgement a dès ses débuts des répercussions importantes sur la concentration foliaire en manganèse, d'une façon encore plus nette que pour l'azote. Ainsi, dans l'expérience correspondant aux figures 5 à 7 , la teneur des aiguilles en $\mathrm{Mn}$ était, au 20 jour de l'engorgement, de $0,11 \mathrm{p} .1000$ pour 
les témoins et de 0,04 p. 1000 pour les plants ennoyés, la différence s'accentuant pour des engorgements de plus longue durée. Ce phénomène pourrait pcut-être être utilisé dans le diagnostic de l'engorgement, bien qu'il n'apparaisse plus trois mois après le ressuyage et qu'il ne permette guère de comparer valablement que des stations sur substrat identique. Un barbotage d'air ou le renouvellement quotidien de l'eau n'améliorent pas la concentration de $\mathrm{Mn}$ dans les aiguilles.

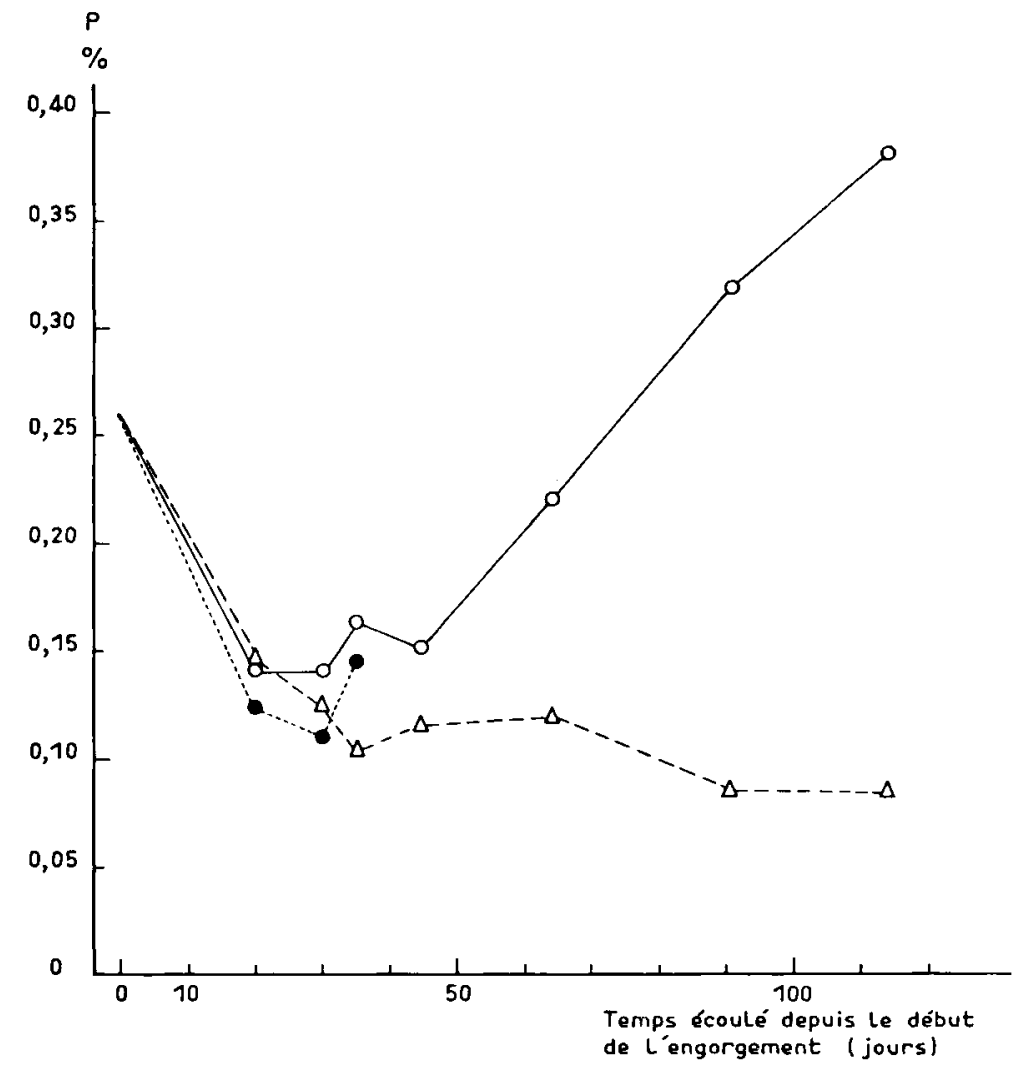

Fig. 6

Influence de l'engorgement de surface sur la teneur en phosphore des aiguilles Influence of surface water logging on phosphorus needles content

Légende :

Témoin non engorgé.

No water-table.

...... Nappe en surface.

Surface water-table.

- - $\triangle$ Nappe en surface avec barbotage d'air.

Surface water-table with air bubbling. 


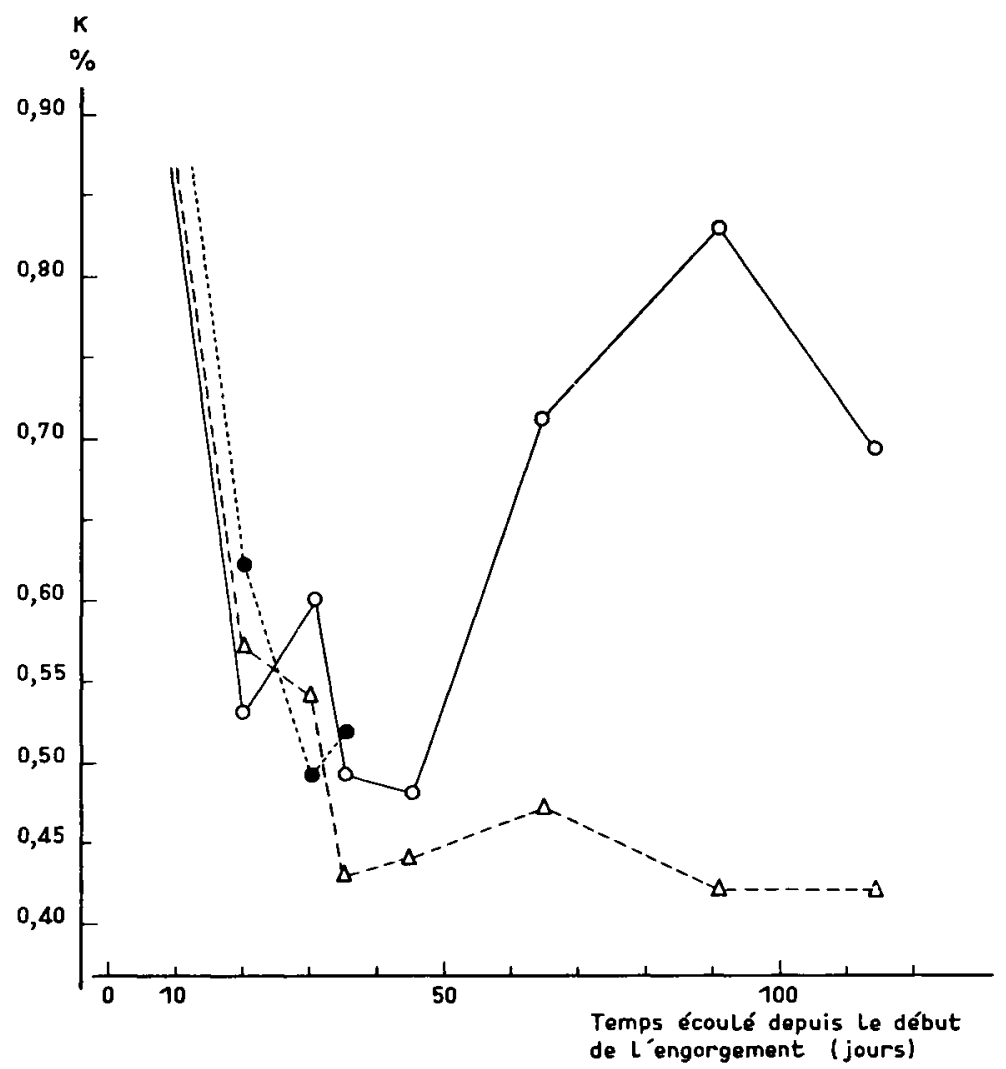

FIG. 7

Influence de l'engorgement de surface sur la teneur en potassium des aiguilles Influence of surface water logging on potassium needles content

Légende :

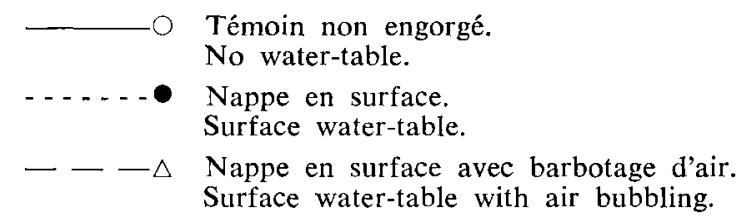

3.32. Influence d'une amélioration de la nutrition minérale (et de la teneur en certaines hormones) sur le comportement des plants

II est nécessaire de signaler, en préambule, un phénomène toujours constaté dans ces expériences : les plants témoins, élevés en milieu non engorgé, réagissent très nettement à une fertilisation minérale, alors qu'en conditions naturelles (forêt) l'apport d'engrais n'est pas efficace sur ce type de sol relativement riche; la fertilisation dans les vases de végétation est toujours efficace, que l'on ait, sous le mull mésotrophe, 
un horizon $\mathrm{A}_{2}$ limoneux ou un sable grossier. On suppose qu'en forêt les conditions climatiques constituent le facteur limitant essentiel de la croissance des plants, alors que le microclimat favorable de la serre permet à l'amélioration de la nutrition minérale, consécutive à la fertilisation, de s'exprimer. C'est principalement l'apport de phosphore qui est efficace, l'azote n'ayant une petite action supplémentaire que lorsque la nutrition phosphorée est améliorée.

\subsection{Fertilisation du sol peu avant l'engorgement}

Elle se traduit d'une façon générale par une amélioration très nette du taux de survie et par une certaine augmentation de longueur de la pousse terminale de l'année (la comparaison des croissances n'est cependant valable que tant que le pourcentage de mortalité du témoin engorgé n'est pas trop important).

Mais, contrairement au cas des plants non engorgés, c'est de loin l'azote qui a l'action la plus efficace. Ainsi, l'une des expériences a fourni les résultats suivants :

\begin{tabular}{|c|c|c|c|}
\hline & \multicolumn{2}{|c|}{ Plants en bonne santé $(\%)$} & \multirow{2}{*}{$\begin{array}{l}\text { Pousse depuis } \\
\text { l'engorgement } \\
\text { at } 25^{\circ} \text { jour }(\mathrm{cm})\end{array}$} \\
\hline & $\begin{array}{c}25^{\mathrm{e}} \text { jour } \\
\text { d'engorgement }\end{array}$ & $\begin{array}{c}32^{\prime \prime} \text { jour } \\
\text { d'engorgement }\end{array}$ & \\
\hline Nappe en surface $T$ & 61 & 17 & 2,8 \\
\hline Nappe en surface PK (1) & 67 & 50 & 2,8 \\
\hline Nappe en surface $N \ldots$ & 89 & 78 & 3,5 \\
\hline Nappe en surface NPK & 94 & 78 & 3,6 \\
\hline Pas de nappe $T$ & 100 & 100 & 3,4 \\
\hline Pas de nappe NPK (1) & 100 & 100 & 5,0 \\
\hline
\end{tabular}

(1) D'autres expériences ont permis de montrer que l'effet favorable de ce traitement est surtout dû̀ à $P$.

Ces résultats sont obtenus sur un sol différent de ceux utilisés habituellement; il s'agit d'un mull acide à texture limono-sableuse, plus pauvre en $\mathrm{P}_{\mathrm{s}} \mathrm{O}_{5}(0,07 \%)$. Avec le mull mésotrophe des autres expériences, l'apport préalable de phosphore n'est favorable dans le cas d'une nappe superficielle que s'il est combiné avec un apport d'azote, sinon il est néfaste : le rapport $\mathbf{N} / \mathbf{P}$ dans les feuilles, dont on connaît par ailleurs l'importance chez les résineux, ne doit donc pas être trop déséquilibré.

Cette fertilisation $\mathrm{N}$ préalable permet de beaucoup améliorer la teneur foliaire en azote des plants engorgés, sans pour autant toujours « rattraper » entièrement celle des plants non engorgés.

La carence azotée créée par la nappe constitue donc une des causes primaires de la mortalité des plants en sol à engorgement de surface; il en est de même, mais à un bien plus faible degré, de la relative pauvreté chimique du sol en phosphore. La cause directe de cette mortalité étant, comme nous avons pu le montrer, le déficit hydrique des parties aériennes, nous pouvons supposer que la carence azotée accentue le déficit hydrique; ceci est en accord avec Mingeau \& RobeI.IN (1972) qui ont montré qu'un déficit en azote diminuait notablement l'absorption de l'eau et induisait un déficit hydrique important chez certains végétaux. Nous avons obtenu ultérieurement confirmation de ces faits (article en cours de rédaction) à l'aide de mesures de pression de sève sur de jeunes rameaux. Quant au déficit en phosphore, on peut penser qu'il diminue encore le peu d'énergie fournie à la racine pendant l'engor- 
gement, en limitant les réactions de phosphorylation; Morizet \& Mingead (1976) ont d'ailleurs montré que la carence en phosphore diminuait, comme celle en azote, l'absorption de l'eau, mais les symptômes sont beaucoup plus longs à apparaître.

La carence azotée provoquée par la nappe est également en partie responsable de la diminution de la croissance aérienne. Mais les déficits de la nutrition minérale n'expliquent qu'une assez faible partie de la différence finale de taille; en effet, bien que les concentrations foliaires en éléments nutritifs soient comparables dans les traitements engorgés et non engorgés qui ont tous deux reçu une fertilisation complète, la pousse des seconds est nettement plus grande, et la différence va en s'accentuant jusqu'à la fin de la croissance de l'année. D'autres phénomènes que la nutrition minérale sont donc en cause; nous avons déjà traité du déficit en eau des tissus : chez des espèces ligneuses, l'engorgement du système racinaire peut ainsi conduire à la fermeture rapide des stomates (Pereira \& Kozlowski, 1977) et à la réduction de la photosynthèse (REGEHR et al., 1975); on peut également penser au déficit en certaines hormones de croissance (cf. § 3.323).

\subsection{Pulvérisation de composés azotés durant l'engorgement}

On peut se demander si la carence en azote provoquée par la nappe ne pourrait pas être mieux (ou du moins aussi bien) combattue par pulvérisation foliaire au cours de l'engorgement que par fertilisation préalable.

Aucun des 3 produits que nous avons appliqués de cette manière n'a d'effet net sur la pousse.

Quant à leurs conséquences sur la survie, elles sont très différentes : l'urée ne modifie pas la mortalité ou bien l'augmente même, les ammonitrates la diminuent un peu, les acides aminés la réduisent très sensiblement. Ainsi, dans l'une des expériences, on a noté les mortalités suivantes, respectivement pour le témoin engorgé, les pulvérisations d'ammonitrates, et les pulvérisations d'acides aminés : le jour du ressuyage (après 42 jours d'engorgement), 44 p. 100,27 p. 100 et 11 p. $100 ; 1,5$ mois après le ressuyage, 96 p. 100,89 p. 100 et 37 p. 100 .

On peut supposer que l'absorption foliaire d'acides aminés permet la migration de composés azotés vers les racines, qui verraient ainsi diminuer leur résistance au transfert de l'eau que l'engorgement tend à augmenter. Ce résultat semble aussi suggérer que c'est sous forme métabolisée (au moins acides aminés) que l'azote favorise l'absorption de l'eau en conditions normales (milieu non engorgé).

\subsection{Pulvérisation de certaines hormones durant l'engorgement}

Il s'agit de produits appartenant à des types d'hormones dont dépend notamment la croissance de la plante : d'une part une gibbéreline, l'acide gibberelique, d'autre part une cytokinine, la benzyladénine.

L'engorgement du sol a pour conséquence de réduire fortement les teneurs en ces deux types d'hormones, au moins chez certains végétaux. Une pulvérisation foliaire d'acide gibberelique ou de benzyladénine permettrait alors de protéger la plante contre les effets de l'engorgement, et en particulier d'améliorer sensiblement la croissance (REID \& Rallton, 1974). Cependant, dans certains cas ces applications n'ont pas d'effet favorable, au contraire, notamment lorsqu'il y a engorgement avec forte ETP (COHEN, 1975). 
Nos expériences n'ont pas donné de résultat positif : les pulvérisations n'ont entraîné aucune différence significative de comportement par rapport au témoin engorgé. On peut donc supposer que les déficits éventuels en ces deux types d'hormones ne sont pas à l'origine du mauvais comportement de l'Epicéa dons nos expériences avec nappe superficielle.

\section{4. - Le développement du système racinaire}

C'est évidemment par l'intermédiaire de son action sur les racines que la nappe modifie le comportement des parties aériennes.

Nous avons vu que le fonctionnement du système racinaire était alors perturbé, notamment l'absorption d'éléments minéraux et d'eau; on suppose que sa morphologie est également affectée.

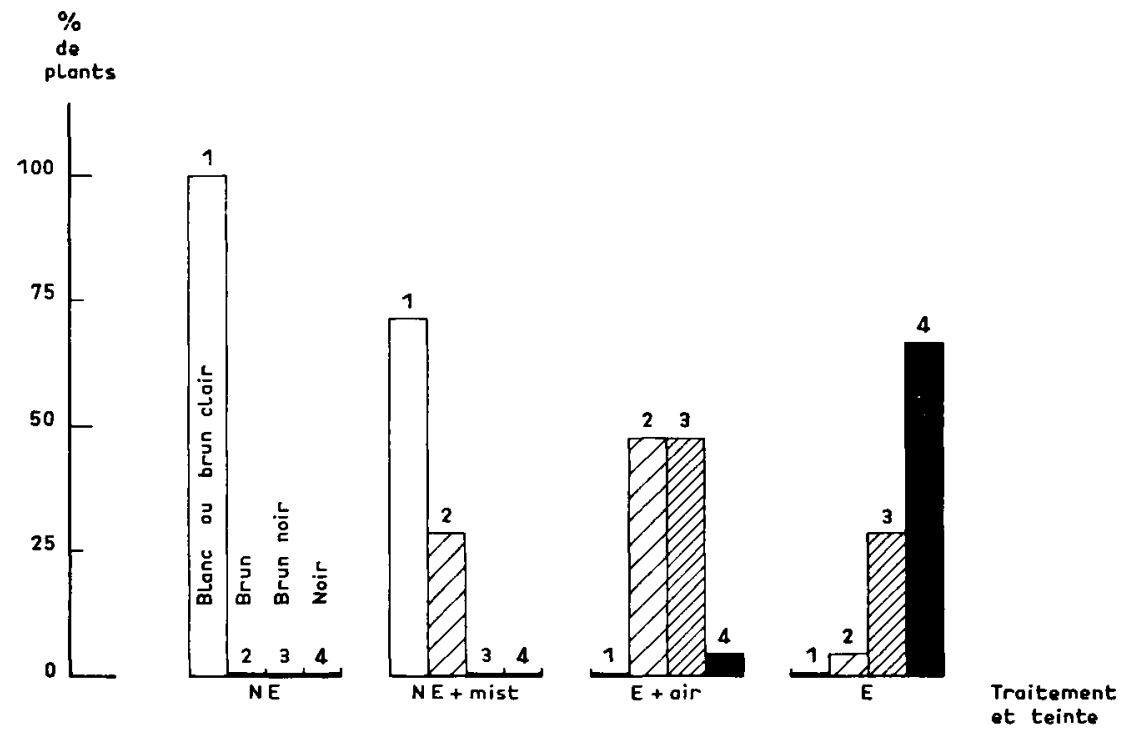

FIG. 8

Teinte des anciennes racines à la fin de l'engorgement

Color of old roots at the end of water logging

Légende :

NE : Non engorgé.

Without water-table.

E : Engorgé en surface.

With surface water-table.

mist : Nébulisation d'eau sur les feuilles.

Spraying of water on needles.

air : Barbotage permanent d'air dans la nappe.

Permanent air bubbling into the water-table. 


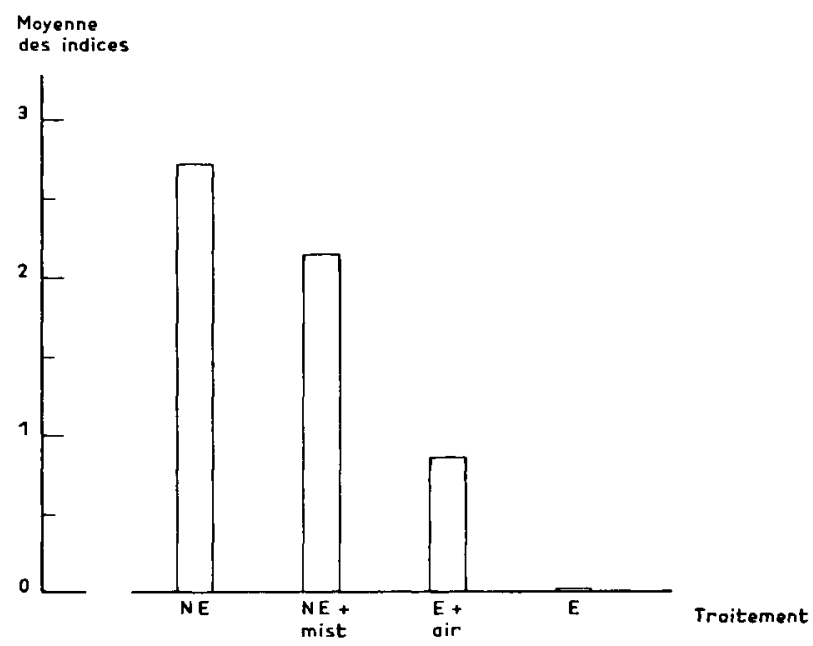

FIG. 9

Densité des nouvelles racines longues à la fin de l'engorgement Density of the new long roots at the end of water logging

\section{Légende :}

Indice pour chaque plant :

Index for each plant :

0 : Pas de racine longue nouvelle.

No new long root.

1 : De 1 à 5 racines longues nouvelles.

1 to 5 new long roots.

2 : De 6 à 10 racines longues nouvelles.

6 to 10 new long roots.

3 : Plus de 10 racines longues nouvelles (NE est donc proportionnellement sousestimé).

More then 10 new long roots.

Se reporter également à la légende de la fig. 8.

See also legend of fig. 8.

\subsection{A la fin d'un engorgement d'assez longue durée}

Nous avons représenté sur les figures 8 à 10, à titre d'exemple, plusieurs variables caractérisant l'état de l'enracinement des plants après 43 jours d'engorgement. Les anciennes racines sont facilement reconnaissables car, avant le repiquage, elles ont ont été «habillées» à une longueur uniforme (environ $8 \mathrm{~cm}$ ) ; elles ne s'agrandissent plus ensuite à partir de leur extrémité.

On peut constater l'influence défavorable d'une augmentation (par nébulisation : $\mathrm{NE}+$ mist) de la teneur en eau du sol, donc d'une diminution de sa porosité à l'air, par rapport à un sol maintenu à la capacité au champ (NE). 


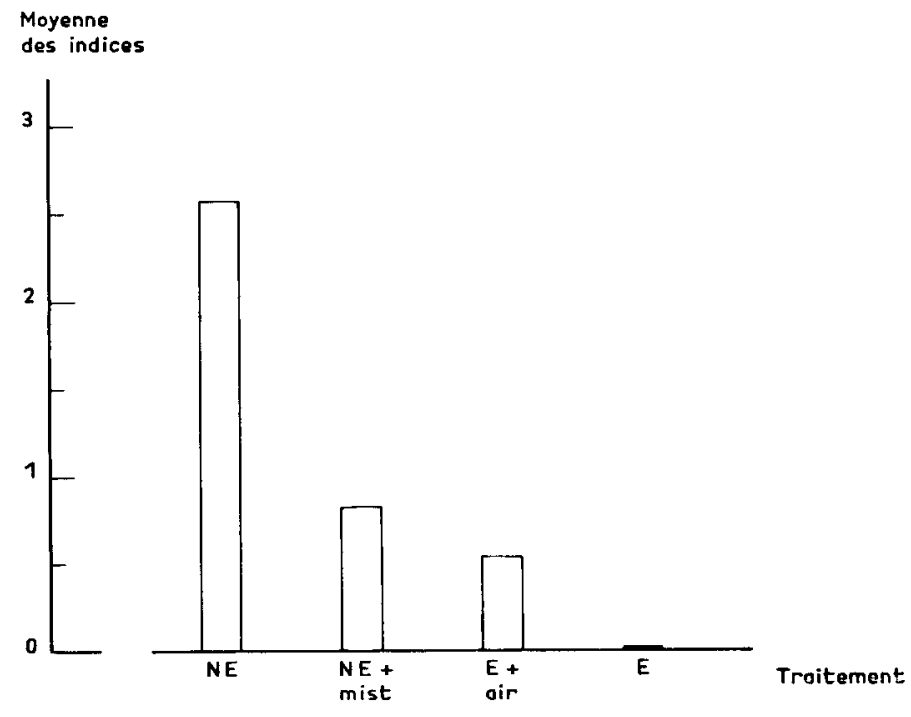

FIG. 10

Densité des racines courtes $(<1 \mathrm{~cm})$ nouvelles prenant naissance sur les racines longues (indice qualitatif pour chaque plant : de 0 à 3 )

Density of new short roots $(<1 \mathrm{~cm})$ growing from the long roots (qualitative index for each plant : from 0 to 3 )

Se reporter également à la légende de la fig. 8.

See also legend of fig. 8.

Il apparaît d'ailleurs sur ces figures que, d'une manière générale, les différentes variables étudiées dépendent directement de l'approvisionnement en air du système racinaire. On constate qu'en présence d'une nappe stagnante (traitement $E$ ), les anciennes racines sont souvent toutes nécrosées, et qu'il n'y a jamais formation de nouvelles racines. Dans les autres traitements, l'excès d'eau diminue plus la densité des nouvelles racines longues que celles des nouvelles racines courtes $(<1 \mathrm{~cm})$.

Par ailleurs, le taux de survie finale (en automne) pour les différents traitements de cette expérience est en rapport avec les variables étudiées, notamment avec la teinte des anciennes racines lors du ressuyage : il est globalement lié à la proportion des racines blanches et brunes par rapport à l'ensemble des racines.

D'autres expériences confirment, en les généralisant, les résultats de celle-ci. Le classement des traitements pour chacune des variables qui caractérisent l'enracinement est en accord avec le potentiel redox de la nappe. Ainsi, des apports de $\mathrm{NO}_{3}-$ conduisent à un enracinement intermédiaire entre le traitement à nappe stagnante et celui qui comporte un barbotage d'air; la suppression initiale de tout horizon organique entraîne à peu près les mêmes conséquences.

Nous avons par ailleurs constaté la formation sur les plants engorgés (en général pas sur les témoins) de deux types d'organes constituant une adaptation à l'hydromorphie chez les espèces ligneuses (GILl, 1970 ; Hook \& Scholtens, 1978) : 
- d'une part des racines, que l'on ne peut pas qualifier d'adventives au sens strict, mais qui prennent naissance un peu au-dessous du collet et sont sub-horizontales et de teinte blanchâtre; elles se développent donc dans un milieu à potentiel redox plus élevé que le reste du système racinaire. On n'en observe qu'assez rarement sur les plants du témoin engorgé, assez souvent (de 0 à plusieurs par plant) si la nappe a bénéficié d'une amélioration de son potentiel redox (barbotage d'air, etc.),

- et surtout, au niveau du collet (mais pas sur les racines), de lenticelles fortement hypertrophiées et souvent proéminantes, permettant une entrée d'air qui serait en principe transféré vers les parties plus profondes du système racinaire; il y en a généralement quelques-unes sur chaque plant lorsque la nappe ne bénéficie pas d'une amélioration de son potentiel redox, nettement plus dans le cas contraire. D'ailleurs, l'aspect extérieur d'un plant, dans un traitement engorgé donné, est très lié à la présence et l'abondance de ces lenticelles hypertrophiées.

\subsection{A l'automne (après engorgement de printemps)}

Parmi les plants ayant subi au printemps une nappe stagnante, peu survivent encore à l'automne. On constate que le système racinaire de ces derniers s'est peu développé au cours de l'été ; ils possèdent quelques racines longues nouvelles, sur lesquelles prennent naissance des racines courtes peu denses. Mais ces quelques plants qui ont résisté montrent une proportion de racines «adventives» (au sens large) et un nombre de lenticelles hypertrophiées bien supérieur à ce qu'on pouvait observer en moyenne lors du ressuyage; cela tend à confirmer le rôle favorable, pour la survie des plants, de ces organes d'adaptation.

L'amélioration du potentiel redox de la nappe au printemps (barbotage d'air, etc.) permet aux plants de posséder à l'automne un système racinaire d'assez bel aspect; le retard considérable qu'ont les plants à nappe stagnante par rapport au témoin non engorgé est supprimé, dans ce cas, pour nettement plus de la moitié, et cela pour les 3 variables observées alors : longueur de l'enracinement, densité de racines longues, densité de racines courtes.

Pour comparer alors quantitativement les différents traitements, nous avons retenu le poids des racines fines $(<1 \mathrm{~mm}$ de diamètre), qui varie moins dans un même traitement en fonction de l'individu que le poids total du système racinaire.

Ainsi, dans l'expérience correspondant aux figures 8 à 10, les poids secs moyens des racines fines d'un plant sont à lautomne les suivants : non engorgé : 2,30 $\mathrm{g}$ nappe «oxygénée 》: $1,58 \mathrm{~g}$ - non engorgé + pulvérisation foliaire d'eau jusqu'à l'automne : 0,98 g - nappe stagnante : $0,31 \mathrm{~g}$ - nappe stagnante (au printemps) + pulvérisation foliaire jusqu'à l'automne : $0,17 \mathrm{~g}$.

La différence de poids sec entre ces deux derniers traitements s'explique par le fait que la pulvérisation foliaire a permis la survie de presque tous les plants sans pour autant améliorer leur enracinement, alors que dans les traitements sans pulvérisation, seuls les plants à enracinement (relativement) le meilleur ont pu survivre au cours de l'été.

Les autres expériences confirment ces résultats. On y constate de plus que la présence de matière organique, qui était néfaste à l'enracinement durant la période d'engorgement, est très utile après le ressuyage, tout au moins pour le développement des racines longues (donc le poids sec du système racinaire) : en présence d'un horizon 
organique, l'enracinement «long» des plants destinés à survivre rattrape, puis dépasse nettement, celui des plants installés dans un substrat exempt de matière organique. Il n'en est pas de même, par contre, pour les racines courtes (qui constituent, semble-t-il, des ébauches de mycorhizes) : l'écart qui existait entre les deux traitements à la fin de la période de ressuyage demeure.

Revenons à l'expérience (d'où a été tirée la figure 1) dans laquelle un engorgement de surface au printemps entraînait une importante mortalité, et affectait la croissance des plants, s'il était remplacé, en été, par une nappe affleurant à $-14 \mathrm{~cm}$, alors qu'un sol beaucoup plus desséché au cours de l'été était nettement plus favorable. Or, l'aspect extérieur des plants encore vivants à l'automne est bien en rapport avec le poids sec de l'ensemble de leurs racines fines : de 2,0 à 3,1 g pour des plants en bonne santé, de 0,7 à $1,6 \mathrm{~g}$ (sauf une exception) pour des plants plus ou moins dépérissants, et cela indépendamment du traitement. On suppose donc que lorsque les racines ont été fortement affectées au printemps, la régénération du système racinaire pendant l'été nécessite des conditions d'oxygénation qu'une nappe maintenue à $-14 \mathrm{~cm}$ ne permet en général pas (il s'agissait dans cette expérience, rappelons-le, de plants installés depuis plus d'un an au moment de l'engorgement).

\section{4. - Conclusion}

Une nappe engorgeant le sol jusqu'en surface en période de croissance aérienne constitue toujours un milieu défavorable, même si elle est relativement aérée, car la diffusion de l'oxygène nécessaire à la respiration des racines est extrêmement lente dans l'eau.

Ses conséquences au niveau du comportement des plants dépendent cependant beaucoup d'une part de son potentiel d'oxydo-réduction, qui est conditionné, en forêt, par les données topographiques, morphologiques, climatiques et édaphiques, et d'autre part des possibilités de diffusion de l'oxygène vers les racines, sur lesquelles interviennent certaines propriétés du sol (texture, structure). L'ensemble de ces variables détermine en effet le flux d'oxygène.

La survie des plants, leur croissance et, dans une certaine mesure, leur nutrition minérale, dépendent globalement des mêmes facteurs du milieu, par l'intermédiaire de l'état et du fonctionnement des racines, mais elles ne sont pas affectées par les mêmes niveaux de ces facteurs.

C'est l'année même de l'installation des plants que la nappe est la plus défavorable; nous avons considéré principalement ce cas.

Une nappe stagnante provoque la nécrose des racines existantes et empêche pratiquement la formation de nouvelles racines. La mortalité des plants apparaît liée à cette nécrose; elle débute en général, dans nos conditions expérimentales, 2 à 3 semaines après le début de l'engorgement, puis elle atteint rapidement la grande majorité des plants, bien qu'extérieurement elle ne se manifeste pas immédiatement. En effet, cette mortalité des parties aériennes est, au moins sur les substrats assez riches, très répandus dans l'Est de la France, qui nous intéressent, la conséquence d'un déficit hydrique dans leurs tissus : après une certaine durée d'engor- 
gement, il semble que l'absorption active d'eau ne s'effectue plus, mais que le plant reste en sursis grâce à une certaine absorption passive; le ressuyage accélère alors fortement la mortalité. Une pousse de l'année plus avancée ou une température plus élevée durant l'engorgement entraînent une plus forie mortalité des plants, car elles augmentent les besoins en oxygène, donc le déficit d'alimentation en eau.

Sur le plan de la nutrition, la conséquence essentielle de l'engorgement est une forte carence en azote, élément qui niest sans doute alors pratiquement jamais absorbé ; cette carence (que nous tenterons d'expliquer dans une publication prochaine) contribue à accélérer lapparition et l'évolution de la mortalité, sans doute par une accentuation du déficit hydrique; en effet, l'insuffisance respiratoire des racines entraîne une diminution de la perméabilité racinaire, le phénomène étant d'autant plus accusé qu'il se prociuit en conditions déficientes en azote (MorızeT \& Mingeau, 1976). Des déficits du sol en phosphore, et peut-être dans d'autres éléments minéraux, favorisent également la mortalité.

La croissance aérienne est très affectée par une nappe de surface, même après le ressuyage; il n'y a, en particulier, pas de formation de pousses d'août. Un engorgement de peu de jours (trois, par exemple) a déjà une certaine influence. La carence azotée et le déficit hydrique créés par l'engorgement contribuent à ce phénomène, mais d'autres causes sont sans doute plus déterminantes.

Une période de ressuyage de quelques jours au cours de l'engorgement, mais sans modifier sa durée totale, améliore survie et croissance des plants.

Le comportement des jeunes plants est nettement plus satisfaisant lorsque le potentiel d'oxydo-réduction au niveau des racines est plus élevé : texture grossière par rapport à texture fine, teneur faible en matière organique (un certain taux de matière organique est cependant bénéfique lorsqu'il permet d'améliorer nettement la structure en surface), nappe plus oxygénée, repiquage «horizontal des racines. Ces améliorations diminuent la nécrose des anciennes racines et permettent la formation de quelques racines nouvelles; comme conséquence, la survie est souvent très nettement améliorée, la croissance aérienne l'est également un peu, mais la carence azotée demeure pratiquement aussi importante.

Les plants sont beaucoup plus résistants si lengorgement de surface n'intervient qu'un an ou plus après leur installation. Une mortalité importante apparaît alors si le sol est maintenu au cours de l'été à une humidité assez forte (nettement supérieure à la capacité au champ), mais pas s'il est normalement ressuyé ; le système racinaire, très affecté par la nappe de printemps, ne peut se régénérer correctement qu'en présence d'une bonne teneur en air.

L'Epicéa commun est une espèce relativement résistante à l'engorgement, donc à l'hypoxic au niveau de son système racinaire; certaines particularités morphologiques observées dans cette étude peuvent sans doute être considérées comme des adaptations à ces conditions défavorables : formation de quelques racines adventives, et surtout hypertrophie spectaculaire des lenticelles du collet, qui peuvent en principe permettre une entrée d'air qui serait transféré ensuite vers les racines. La survie des plants apparaît liée à cette adaptation.

Sur le plan pratique, il apparaît utile biologiquement (mais économiquement?) de drainer, avant plantation d'Epicéa, les sols où il $\mathrm{y}$ a risques d'engorgement de surface durant la période de végétation. Et surtout, il est nécessaire de disposer de 
plants bien nourris en pépinière, grâce à une fertilisation équilibrée. Des apports d'engrais azotés et éventuellement complets seraient également utiles après plantation en forêt, à un moment où les racines sont actives, mais où la nappe est absente ou du moins profonde (assez tôt à l'automne?).

Reçu pour publication en octobre 1980.

\section{Summary}

Behaviour of young plants of Norway Spruce in soil with seasonnal surface water logging : influence of different environmental factors

This article synthesizes several experiments realized in controlled conditions, with plants transplanted when two years old; the water logging generally takes place the same year than transplanting. The soil used, taken from hydromorphic sites in east of France, is chemically relatively rich.

The influence of different factors on plants mortality and growth was studied, particularly : duration of the water logging, temperature, oxidoreduction potential, soil characteristics.

The water deficit of aerial parts of the plants is the real cause of their death on the soil used. Thus, a very frequent spraying of water on the needles stops the dying. Remowing of a long remaining water-table speeds up the dying, probably because it suppresses sumle passive water absorption.

Old roots aspect and new roots density are dependent on air supply possibilities in the rhizosphere. The final survival rate is in connection with these characteristics of the root system and with the development of organs that can be considered as adaptations : particularly very enlarged lenticels on the collar.

Water logging induces an important nitrogen deficit into the needles, which begins very soon after the installation of water-table, even if it is somewhat oxygenated. Foliar manganese content is also greatly reduced. Phosphorus and potassium deficits begin later.

Nitrogen deficiency due to the water-table is one of the main causes of plants mortality. Some soil deficit in phosphorus gives somewhat less important mortality. Nitrogen spraying on needles during water logging are much more efficient if one uses amino-acids than ammonium nitrate or urea.

\section{Références bibliographiques}

Cohen D., 1975. Hormonal responses to environmental stress. A review. Procecd. Agron. Soc. New Zealand, 5, 55-57.

Decourt N.. Levy G., 1974. La productivité de l'Epicéa commun sur les sols hydromorphes de Lorraine : mesure et caractéristiques générales. Ann. Sci. forest., 31 (2), 71-82.

Gill C.J., 1970. The flooding tolerance of woody species. A review. For. abstr., 31 (4), 671-688.

Heller R., 1969. Biologie végétale. II - Nutrition et métabolisme. Masson et $C^{i \text {, }}$, Paris, $578 \mathrm{p}$.

Hook D., Scholtens J., 1978. Adaptations and flood tolerance of tree species, pp. 299-331, in "Plant life in anaerobic environments », D. Hook and R. Crawford editors, Ann. Arbor Science Publishers, 564 p. 
Kramer P.J., 1969. Plant and soil water relationships : a modern synthesis, Mc Graw-Hill Book Company, New York, 482 p.

LEVY G., 1971. Influence de l'engorgement de printemps et de la sécheresse d'été sur le comportement de jeunes plants d'Epicéa. Ann. Sci. forest., 28 (4), 403-423.

Levy G., 1972. Premiers résultats concernant deux expériences d'assainissement du sol sur plantations de résineux. Ann. Sci. forest., 29 (4), 427-450.

LEVY G., 1978. Nutrition et production de l'Epicéa commun adulte sur sols hydromorphes en Lorraine; liaisons avec les caractéristiques stationnelles. Ann. Sci. forest., 35 (1), 33-53.

Mingeau M., Robelin M., 1972. Le transfert de l'eau dans la plante : action particulière de la nutrition azotée. Ann. agron., 23 (4), 419-427.

Morizet J., Mingeau M., 1976. Influence des facteurs du milieu sur l'absorption hydrique. Etude effectuée sur Tomate décapitée en exsudation. Ann. Agron., 27 (2), 183-205 et 27 (3), 333-342.

Pereira J.S., Kozlowsli T.T., 1977. Variations among woody angiosperms in response to flodding. Physiologia plant, 41, (3), 184-192.

Regehr D.L., Bazzaz F.A., Boggers W.R., 1975. Photosynthesis, transpiration and leaf conductance of Populus deltoides in relation to flooding and drought. Photosynthetica, 9 (1), 52-61.

Reid D.M., Railton I.D., 1974. The influence of benzyladenine and gibberellin content of shoots on the growth of waterlogged Tomato plants. Plant Sci. letters, 2, 151-156.

Sanderson P.L., Armstrong W., 1978. Soil waterlogging, root rot and conifer windthrow : oxygen deficiency or phytotoxicity? Plant Soil, 49 (1), 185-190. 